\title{
COOLING-INDUCED STRUCTURES IN COLLAPSAR ACCRETION DISKS
}

\author{
A. Batta ${ }^{1}$ and W.H. Lee ${ }^{1}$
}

\begin{abstract}
The collapse of massive rotating stellar cores and the associated accretion is thought to power long Gamma ray bursts. The physical conditions make neutrino emission the main cooling agent in the flow. We have carried out an initial set of calculations of the collapse of rotating polytropic cores in three dimensions, making use of a pseudo-relativistic potential and a simplified cooling prescription. We focus on the effects of self gravity and cooling on the overall morphology and evolution of the flow for a given rotation rate in the context of the collapsar model. For the typical cooling times expected in such a scenario we observe the appearance of strong instabilities on a time scale $t_{\text {cool }}$ following disk formation. Such instabilities and their gravitational interaction with the black hole produce significant variability in the obtained accretion rates, which would translate into luminosity variations when a more realistic neutrino cooling and luminosity scheme is implemented in future work.
\end{abstract}

\section{Introduction}

To date it is generally accepted that Gamma ray bursts (GRBs) are the result of cataclysmic events involving Neutron stars (NSs) or black holes (BHs). More over, long GRBs are generally associated with actively star forming galaxies and sometimes with a Supernova (SN) type Ib or Ic, taking place at the same time and place. A review by Woosley \& Bloom (2006) shows the existing evidence at the time for the link of long GRB at low redshift with type Ic SNe.

In this work we will consider the collapsar model (Woosley 1993) to in which the formation of a GRB follows from the collapse of a pre-supernova (PreSN) star. In such a scenario, a BH is formed from the star's Fe core while the outer rotating layers collapse and form an accretion disk around the BH. With the high temperatures and densities expected in the disk $\left(T \geq 10^{10} \mathrm{~K}, \rho \geq 10^{9} \mathrm{~g} \mathrm{~cm}^{-3}\right)$, neutrino emission becomes the main cooling mechanism. These neutrinos provide the main

\footnotetext{
1 Instituto de Astronomía, Universidad Nacional Autónoma de México, Apdo. postal 70-264, Ciudad Universitaria, D.F., México; e-mail: abatta@astro.unam.mx; wlee@astro.unam.mx
} 
cooling mechanism, and may contribute, along with magnetically powered outflows, to power the GRB.

Besides the work by MacFadyen \& Woosley (1999), (the first one to explore the collapsar scenario in a 2D hydrodynamic simulation) a body of work has been carried out in 2D with improved physics (realistic EOS, MHD (Proga et al. 2003), or improved neutrino cooling prescriptions (López-Cámara et al. 2009) but, they have not properly accounted for instabilities that may come from the combination of self gravity and cooling processes. For the 3D studies that have been carried out (Rockefeller et al. 2006; Taylor et al. 2011), we do not yet have a complete understanding of the importance of structure formation in the accretion flow and or heating/cooling mechanisms. Here we focus on the study of the effects of self gravity and cooling on 3D simulations of the collapse of a rotating polytropic envelope onto a $\mathrm{BH}$ in the context of the collapsar model.

\section{Initial conditions and input physics}

In the context of the collapsar model, we studied the collapse and accretion of $2.5 M_{\odot}$ rotating polytropic envelopes $(\gamma=5 / 3)$ onto a $2 M_{\odot} \mathrm{BH}$ fixed at the center of the distribution. All numerical simulations were made using a modified version of GADGET-2 (Springel 2005).

The $2.5 M_{\odot}$ spherical polytropic envelope is located from $r_{i n t}=844.69 \mathrm{~km}$ to $R_{s}=1715.7 \mathrm{~km}$, surrounding the $2 M_{\odot} \mathrm{BH}$ at the center of the distribution. Such a polytropic envelope was given a solid body rotation just below breakup, which also guaranteed a circularization radius $r_{c}=12.5 r_{a c c}$ close to the accretion radius $r_{a c c}$. We will consider a Paczynski-Wiita pseudo-potential (Paczynski \& Wiita 1980) to account for the most important general relativistic effects determining the motion of matter near a non-rotating $\mathrm{BH}$. The $2 M_{\odot} \mathrm{BH}$ particle will be artificially fixed at the origin by canceling the forces acting on it. The Paczynski-Wiita potential will reproduce exactly, the location of the innermost stable circular orbit $\left(r_{i s c o}=3 r_{g}=6 G M_{B H} / c^{2}\right)$ of a Schwarzchild BH, which will be considered as our accretion radius $\left(r_{a c c}=3 r_{g}\right)$.

We adopted a simplified cooling prescription based on a fixed cooling time $t_{\text {cool }}$ dependent on the dynamical time scale of the accretion disk $t_{d i s k} \simeq 10^{-1} \mathrm{~s}$, formed around the $\mathrm{BH}$.

$$
t_{c o o l}=\beta t_{d i s k}, \quad \frac{d u}{d t}=-u / t_{c o o l} .
$$

The efficiency parameter $\beta$ determines how many times the gas must orbit the $\mathrm{BH}$ before it gets significantly cooled. To account for a cooling as efficient as neutrinos, we explored the case where $t_{\text {cool }}$ is close to the neutrino cooling time scale $t_{\nu}$. Therefore, we estimated the neutrino cooling time scale $t_{\nu}=u / q_{\nu}$ for the densities and temperatures expected near the BH. By considering an approximated EOS composed by relativistic non-degenerate electron-positron pairs and ionized $\mathrm{H}$, as well as a neutrino cooling prescription $q_{\nu}$ accounting for pair annihilation and electron-positron capture (Narayan et al. 2001), the neutrino cooling time scale $t_{\nu}$ can be evaluated as a function of $T$ and $\rho$ from the internal energy and 
cooling rate, respectively:

$$
u=\frac{3}{2} \frac{k T \rho}{\mu m_{p}}+\frac{11}{4} a T^{4}, \quad q_{\nu} \simeq 5 \times 10^{33} T_{11}^{9}+9.0 \times 10^{23} \rho T_{11}^{6} \mathrm{erg} \mathrm{cm}^{-3} \mathrm{~s}^{-1} .
$$

This turns into a neutrino cooling time scale ranging from a few seconds to $\sim 10^{-4} \mathrm{~S}$ for $\rho=10^{10}$ to $10^{12} \mathrm{~g} \mathrm{~cm}^{-3}$ and $T=10^{10}$ to $10^{11} \mathrm{~K}$. Therefore, by using $t_{\text {cool }}=$ $1.23,0.24,0.12,0.061,0.012,0.006 \mathrm{~s}$ in our cooling scheme $(2.1)$, we will explore the importance of the neutrino cooling efficiency in structure formation.

\section{Results and discussion}

By looking at the $\mathrm{BH}$ mass accretion rate $\dot{M}=d M / d t$, and the total energy loss rate $L_{c}=\int d u / d t d V$ obtained for three different cooling schemes we will obtain information on the general changes in the behavior of the accretion flow with increasing cooling efficiency. Figure 1 shows the mass accretion rates (left panel) and the energy loss rates $L_{c}$ (right panel) for models with $t_{\text {cool }}=0.24,0.061,0.012 \mathrm{~s}$, (blue, black and orange lines respectively). Figure 1 shows that as the cooling efficiency increases, the variations in both $\dot{M}$ and $L_{c}$ become more intense. Moreover, intense increases in $\dot{M}$ and $L_{c}$ appear to coincide in time. This is possibly related to the fact that overdense regions become hotter and thus more intense emitters, and that the spiral structures they are associated with transport angular momentum more efficiently, raising the accretion rate. This clearly deserves quantification through further study.
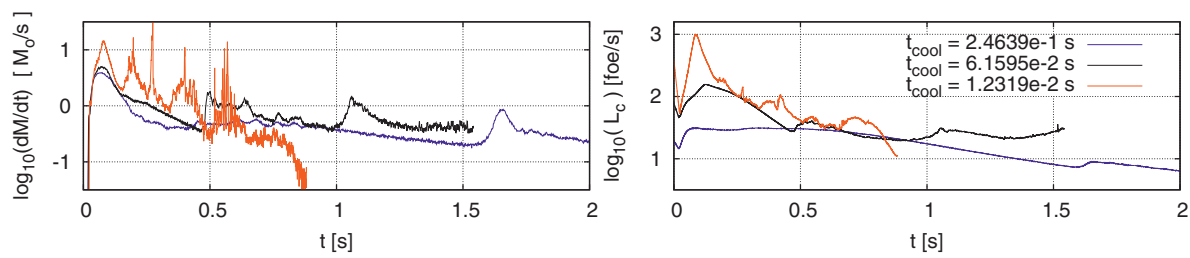

Fig. 1. Logarithm of the $\mathrm{BH}$ accretion mass rate $\dot{M}$ in solar masses per second (left panel) and Logarithm of the energy loss rate $L_{c}$ in foes per second (right panel), for models with $t_{\text {cool }}=1.23,0.24,0.12,0.061,0.012,0.006 \mathrm{~s}$, (red, blue, pink, black, orange and gray lines respectively). Intense variations in both $L_{c}$ and $\dot{M}$ coincide in time. 1 foe $=10^{51} \mathrm{erg}$.

In order to obtain information about the formation of non-axisymmetric instabilities at the disk, we performed a Fourier transform of the azimuthal distribution of mass $\Phi_{M}=\int\left[\int \rho(\phi, r, z) d z\right] r d r$ (Zurek \& Benz 1986) defining the amplitude of the mode $m$ by:

$$
C_{m}=\frac{1}{2 \pi} \int_{0}^{2 \pi} e^{i m \phi} \Phi_{M} d \phi .
$$

The relative power $\left|c_{m}\right|^{2}=\left|C_{m}\right|^{2} /\left|C_{0}\right|^{2}$ in the $m$-th mode indicates the intensity of $m$ spiral arms compared to the disk integrated mass. If such spiral structures are present in the disk, they should be visible in density or internal energy maps. Moreover, they should be also visible as unstable regions by plotting the Toomre 
parameter $Q_{T}=\kappa c_{s} /(\pi G \Sigma)$, determined by the surface density $\Sigma$, the local sound speed $c_{s}$ and the local epicyclic frequency $\kappa$.

Figure 2 (left) shows the evolution of the relative power $\left|c_{2}\right|^{2}$ for the model with $t_{\text {cool }}=0.25 \mathrm{~s}$. This mode has the most intense peak of all modes explored $(m=1,2,3,4)$ whose increase begins around $t \simeq 1.5 \mathrm{~s}$, and is essentially the only one present. The peak shown at $t \simeq 1.6 \mathrm{~s}$ coincides in time with the intense variations in Figure 1 of $L_{c}$ and $\dot{M}$, and thus seem to originate from the changes in $\left|c_{2}\right|^{2}$. Consistently with the increase in relative power in $\left|c_{2}\right|^{2}$, in the right panel we can see the formation of only two spiral arms in the Toomre parameter map.
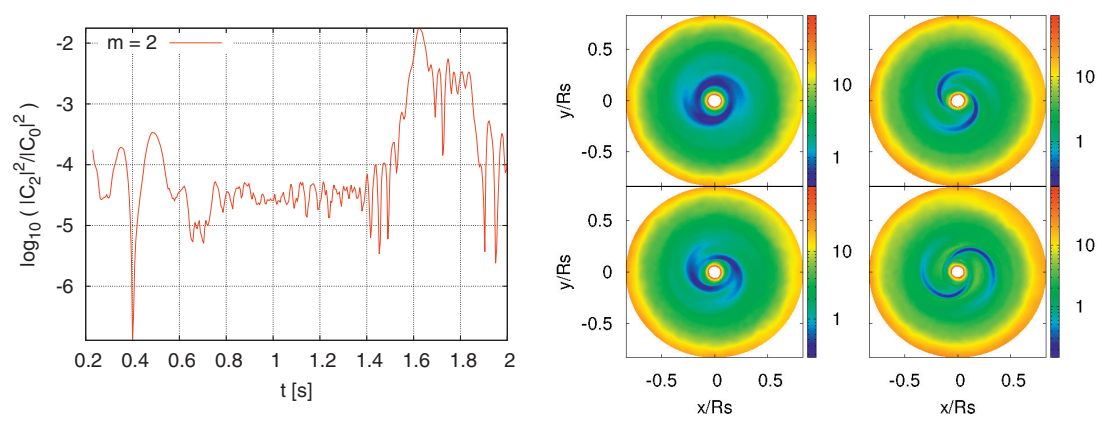

Fig. 2. Relative power $\left|c_{2}\right|^{2}$ for model with $t_{\text {cool }}=0.24 \mathrm{~s}$ (left panel) and evolution of the Toomre parameter $Q$ at times $\simeq 1.6 \mathrm{~s}$ where the azimuthal mode $\mathrm{m}=2$ peaks.

In Figure 3 (left) we see the evolution of the relative power $\left|c_{1}\right|^{2}$ for the model with $t_{\text {cool }}=0.061 \mathrm{~s}$, showing several peaks starting at $t \simeq 0.5 \mathrm{~s}$. The peak at around $t \simeq 0.6 \mathrm{~s}$ in the $m=1$ mode can be seen in the Toomre parameter maps shown in the right panel, where, unlike previously, higher modes are clearly present. We also notice the appearance of gas clumps due to the highly efficient cooling, whose disruption due to close encounters with the $\mathrm{BH}$ are able to induce intense variations in both $\dot{M}$ and $L_{c}$. Such intense variations are noticeable only in the most efficiently cooled models.
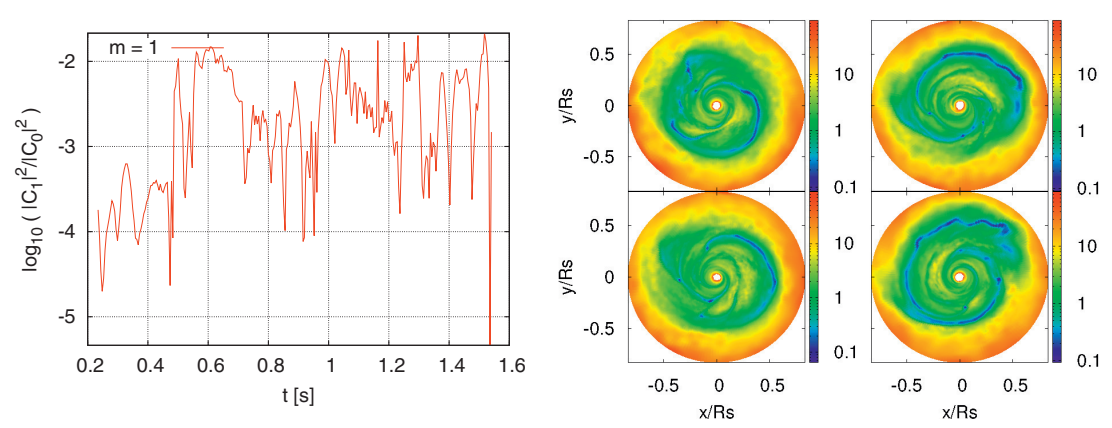

Fig. 3. Relative power $\left|c_{1}\right|$ for model with $t_{\text {cool }}=0.061 \mathrm{~s}$ (left panel) and evolution of the Toomre parameter $Q$ at times $\simeq 0.6 \mathrm{~s}$, right after the first intense increase in all modes. Gas clumps appear due to the high cooling efficiency. 


\section{Summary}

As we found in our simplified collapsar scenario, structure formation at the disk becomes more copious and intense with increasing cooling efficiency. The mass of spiral structures detected with the relative power $\left|c_{m}\right|^{2}$ becomes significantly higher when the cooling parameter $\beta$ is smaller than $\sim 0.6$. Spiral structures detected on the most efficiently cooled models have masses ranging between $0.01 \%-0.1 \%$ of the disk's total mass. This, combined with the formation of massive gas clumps in the disk, indicates that relaxing the assumption that the $\mathrm{BH}$ remains fixed at the origin could lead to interesting behavior. Also, close encounters of gas clumps with the BH can produce significant increases in both the mass accretion and energy loss rates. Therefore, we expect that when more realistic initial conditions and more detailed neutrino cooling are implemented on future work, intense and copious structure may form, altering the neutrino emission itself, as well as the assumed BH-disk symmetric gravitational interaction. Future work will explore the scenario where the $\mathrm{BH}$ is able to move from its original position, as well as the implications of such structure formation events when producing a GRB.

Support from CONACyT (83254 and a graduate fellowship for AB) and the GRB 2012 Symposium LOC, as well as cpu-time at the IA-UNAM cluster Atocatl and at the ICN-UNAM cluster Diable is gratefully acknowledged.

\section{References}

López-Cámara, D., Lee, W.H., \& Ramirez-Ruiz, E., 2009, ApJ, 692, 804

MacFadyen, A.I., \& Woosley, S.E., 1999, ApJ, 524, 262

Narayan, R., Piran, T., \& Kumar, P., 2001, ApJ, 557, 949

Paczynski, B., \& Wiita, P.J., 1980, A\&A, 88, 23

Proga, D., MacFadyen, A.I., Armitage, P., \& Begelman, M., 2003, ApJ, 599, L5

Rockefeller, G., Fryer, C.L., \& Li, H., 2006 [arXiv:astro-ph/0608028]

Taylor, P.A., Miller, J.C., \& Podsiadlowski, P., 2011, MNRAS, 410, 4, 2385

Woosley, S.E., 1993, ApJ, 405, 273

Woosley, S.E., \& Bloom, J.S., 2006, ARA\&A, 44

Springel, V., 2005, MNRAS, 364, 1105

Zurek, W.H., \& Benz, W., 1986, ApJ, 308, 123 
